\title{
La relevancia clínica de la sobreexpresión de HER-2 en el cáncer de mama
}

Khosravi Shahi P, Pérez Manga G. La relevancia clínica de la sobreexpresión de HER-2 en el cáncer de mama. An Med Interna (Madrid) 2006; 103104.

El cáncer de mama incluye un grupo heterogéneo de neoplasias con una historia natural diferente. Los estudios más recientes mediante el empleo de la técnica de microarrays del DNA (1), que permite el estudio sincrónico de miles de genes expresados por un tumor, pone de manifiesto la existencia de tres grandes subtipos de carcinoma de mama invasivo desde el punto de vista del perfil genético, con relevancia pronostica y terapéutica (2): luminal (A, B y C), HER-2 positivo y "basallike".

1. El subtipo luminal se caracteriza por una asociación a una alta expresión de receptores estrogénicos (RE) y a los genes regulados por los estrógenos, lo que proporciona una alta tasa de respuesta a la hormonoterapia y una mayor supervivencia.

2. El subtipo HER-2 positivo presenta el fenotipo RE negativo y se asocia con la sobreexpresión de genes relacionados con una mayor agresividad biológica. Este subtipo tiene un peor pronostico, presentando una resistencia relativa a determinados agentes quimioterápicos y al tamoxifeno (3).

3. El subtipo "basal-like" también es denominado el fenotipo triple negativo (receptores estrogénicos, progesterónicos y HER-2 negativos). En la mitad de los casos presentan una sobreexpresión de EGFR, y se asocian con hallazgos histopatológicos de mal pronostico como alto grado, pleomorfismo o un alto índice mitótico. Es un subtipo de mal pronóstico, pero paradójicamente se asocia a una alta tasa de respuesta a la quimioterapia.

El gen HER-2/neu pertenece a la familia de genes que codifican los receptores que tienen por ligando diversos factores de crecimiento. El HER-2 es por tanto una glicoproteína transmembrana de la superfamilia de las glicoproteínas Erb$\mathrm{B}$, que presenta una actividad tirosin quinasa $(\mathrm{TK})(4,5)$. Existen cuatro subtipos de Erb-B: a) Erb-B1 (HER-1) que es más conocido como EGFR (Epidermal Growth Factor Receptor), que se sobreexpresa en diversos tumores como cáncer colorrectal o carcinomas escamosos de cabeza y cuello; b) Erb-B2 (HER-2), que se asocia fundamentalmente a un subtipo especial de cáncer de mama; c) Erb-B3 (HER-3), es la única que carece de la actividad TK; y d) Erb-B4 (HER-4).
La expresión de HER-2 confiere unas características biológicas específicas (tumores de alto grado, pobremente diferenciado, alto índice mitótico, invasión de ganglios linfáticos) (3) al cáncer de mama con relevancia clínica, puesto que el subgrupo de cáncer de mama HER-2 positivo presenta un peor pronóstico. Este subgrupo supone aproximadamente el 15-20\% de los cánceres de mama infiltrantes, de los cuales a su vez el $50 \%$ no expresan receptores hormonales (6). La amplificación del gen HER-2/neu (localizado en cromosoma 17) (7) y la consecuente sobreexpresión de HER-2 se produce en etapas muy precoces del desarrollo tumoral (presente en la fase de carcinoma "in sitú").

El subgrupo de carcinoma de mama HER-2 positivos presenta cierto grado de resistencia al tamoxifeno y a la quimioterapia con esquema CMF (ciclofosfamida, metotrexate y 5Fluoruracilo), lo que obliga a usar quimioterapias con esquemas que incluyan antraciclinas y/o taxanos, así como recurrir a los inhibidores de aromatasa.

Sin embargo, con el desarrollo del anticuerpo monoclonal anti-HER2 denominado Trastuzumab (Herceptin) (8) el pronóstico de este subgrupo puede ser modificado, al combinarlo con la quimioterapia. Trastuzumab ha demostrado su utilidad en los cánceres de mama HER-2 positivo metastáticos, y estudios recientes demuestran que su uso tanto en la neoadyuvancia junto con quimioterpia con esquema paclitaxel seguido de FEC (5-Fluoruracilo, epirrubicina y ciclofosfamida) (9), como en la coadyuvancia se asocia con una mejora de la supervivencia libre de enfermedad (SLE), aunque todavía no se ha podido demostrar un aumento de la supervivencia global. Sin embargo, su uso se asocia con una preocupante toxicidad cardiaca, en un porcentaje variable de $0,5-4,1 \%(6,9)$, en forma de insuficiencia cardiaca sistólica severa (clase funcional NYHA III-IV) por disminución de la fracción de eyección del ventrículo izquierdo.

En los estudio B-31 y N9831, la combinación de AC (doxorrubicina y ciclofosfamida) seguido por trastuzumab con paclitaxel se asoció con una reducción estadísticamente significativa del $33 \%$ en el riesgo de muerte $(\mathrm{P}=0,015)(10)$. Y en el ensayo llevado por Piccart-Gebhart MJ y cols. (6) la 
combinación de quimioterpia y un año de trastuzumab mejoró significativamente la SLE, con un beneficio absoluto de $8,4 \%$ a los dos años.

\section{Bibliografía}

1. Raetz EA, Moos PJ. Impact of microarray technology in clinical oncology. Cancer Invest 2004; 22: 312-20.

2. 7. O'Connell P. Genetic and cytogenetic analyses of breast cancer yield different perspectives of a complex disease. Breast Cancer Res Treat 2003; 78: 347-9.

3. Burstein HJ. The distinctive nature of HER 2 of breast cancers. N Engl $\mathrm{J}$ Med 353; 16: 1652-1654.

4. Yarden Y, Sliwkowski M. Untangling the ErbB signalling network. Nat Rev Mol Cell Biol 2001; 2: 127-37.

5. Gschwind A, Fischer OM, Ullrich A. The discovery of receptor tyrosine kinases: targets for cancer therapy. Nat Rev Cancer 2004; 4: 361-70.

6. Piccart-Gebhart MJ, Procter M, Leyland-Jones B, Goldhirsch A, Untch M, Smith I, et al. Trastuzumab after adjuvant chemotherapy in HER2-
P. KHOSRAVI SHAHI, G. PÉREZ MANGA

Servicio de Oncología Médica. Hospital General Universitario Gregorio Marañon. Madrid positive breast cancer. N Engl J Med 2005; 353: 1659-72.

7. Wooster R, Weber BL. Breast and ovarian cancer. N Engl J Med 2003; 348: 2339-47.

8. Bell R. What can we learn from Herceptin trials in metastatic breast cancer? Oncology 2002; 63: 39-46.

9. Buzdar AU, Ibrahim NK, Francis D, Booser DJ, Thomas ES, Theriault RL, et al. Significantly higher pathologic complete remission rate after neoadjuvant therapy with trastuzumab, paclitaxel, and epirubicin chemotherapy: Results of randomized trial in human epidermal growth factor receptor 2positive operable breast cancer. J Clin Oncol 2005; 23: 1215-21.

10. Romond EH, Perez EA, Bryant J, Suman VJ, Geyer CE, Davidson NE, et al. Trastuzumab plus adjuvant chemotherapy for operable HER2positive breast cancer. N Engl J Med 2005; 353: 1673-84. 